\title{
BMJ Open Treatment approach and survival from glioblastoma: results from a population- based retrospective cohort study from Western Norway
}

\author{
Line Sagerup Bjorland (10 ,1,2 Oystein Fluge, ${ }^{3,4}$ Bjornar Gilje, ${ }^{2}$ \\ Rupavathana Mahesparan, ${ }^{1,5}$ Elisabeth Farbu ${ }^{1,6}$
}

To cite: Bjorland LS, Fluge 0 , Gilje B, et al. Treatment approach and survival from glioblastoma: results from a population-based retrospective cohort study from Western Norway. BMJ Open 2021;11:e043208. doi:10.1136/ bmjopen-2020-043208

- Prepublication history is published online only. To view, please visit the journal online (http://dx.doi.org/10.1136/ bmjopen-2020-043208).

Received 30 July 2020 Revised 23 December 2020 Accepted 05 February 2021

Check for updates

(C) Author(s) (or their employer(s)) 2021. Re-use permitted under CC BY-NC. No commercial re-use. See rights and permissions. Published by BMJ.

For numbered affiliations see end of article.

Correspondence to Dr Line Sagerup Bjorland; line.bjorland@sus.no

\section{ABSTRACT}

Objectives To evaluate treatment and survival from glioblastoma in a real-world setting.

Design and settings A population-based retrospective cohort study from Western Norway.

Participants 363 patients aged 18 years or older diagnosed with glioblastoma between 1 January 2007 and 31 December 2014.

Primary and secondary outcome measures Overall survival and survival rates determined by Kaplan-Meier method, groups compared by log-rank test. Associations between clinical characteristics and treatment approach assessed by logistic regression. Associations between treatment approach and outcome analysed by Cox regression.

Results Median overall survival was 10.2 months (95\% Cl 9.1 to 11.3). Resection was performed in 221 patients $(60.9 \%)$, and was inversely associated with age over 70 years, higher comorbidity burden, deep-seated tumour localisation and multifocality. Median survival was 13.7 months $(95 \% \mathrm{Cl} 12.1$ to 15.4$)$ in patients undergoing tumour resection, 8.3 months $(95 \% \mathrm{Cl} 6.6$ to 9.9$)$ in patients undergoing biopsy and 4.5 months $(95 \% \mathrm{Cl} 4.0$ to 5.1) in patients where no surgical intervention was performed. Chemoradiotherapy according to the Stupp protocol was given to 157 patients (43\%). Age over 70 years, higher comorbidity burden and cognitive impairment were associated with less intensive chemoradiotherapy. Median survival was 16.3 months ( $95 \% \mathrm{Cl} 14.1$ to 18.5 ), 7.9 months $(95 \% \mathrm{Cl} 6.7$ to 9.0$)$ and 2.0 months $(95 \% \mathrm{Cl} 0.9$ to 3.2) in patients treated according to the Stupp protocol, with less intensive chemoradiotherapy and with best supportive care, respectively. Surgical resection (HR 0.61 (95\% $\mathrm{Cl} 0.47$ to 0.79$)$ ) and chemoradiotherapy according to the Stupp protocol (HR 0.09 (95\% Cl 0.06 to 0.15$)$ ) were strongly associated with favourable overall survival, when adjusted for clinical variables.

Conclusions In a real-world setting, less than half of the patients received full-course chemoradiotherapy, with a median survival comparable to results from clinical trials. Survival was considerably worse in patients receiving less intensive treatment. Our results point out a substantial risk of undertreating glioblastoma, especially in elderly patients.
Strengths and limitations of this study

- This population-based study provides knowledge on treatment and survival from glioblastoma in a realworld setting, including the establishment of longterm survival rates.

- To our knowledge, this is the first study to use a standardised score in the assessment of comorbidity burden in patients with glioblastoma.

- Detailed information on treatment and complications were available in all patients, within a common patient record system used throughout the region.

- We included both patients with histologically confirmed glioblastoma and patients with an MRI-based diagnosis to counteract the exclusion of elderly, frail patients and patients with deep-seated tumours where biopsy was considered not feasible.

- Limitations of this study included the lack of molecular analysis and standardised performance assessment.

\section{INTRODUCTION}

Glioblastoma WHO grade IV is the most frequent of the malignant primary brain tumours in adults. ${ }^{12}$ Prognosis is poor, with a median overall survival of approximately 11 months and a 5 -year survival of $<6 \%$ reported from population-based materials. ${ }^{1}{ }^{3}$ Standard diagnostic procedures in patients with primary brain tumours include neuroimaging and histopathological and molecular classification. ${ }^{4}$ However, when clinicians consider a biopsy unsafe or not feasible, for example, in patients with poor functional status or patients harbouring a deep-seated tumour, the diagnosis is based solely on radiological characteristics. Advanced MRI modalities have resulted in a greater ability to differentiate glioblastoma from other intracranial lesions. 56

Gold standard management of glioblastoma is maximal safe resection or biopsy followed by chemoradiotherapy (CRT). ${ }^{4} 7$ 
The implementation of the Stupp protocol, that is, radiation therapy given as $60 \mathrm{~Gy}$ in 2 Gy fractions with concomitant temozolomide (TMZ) followed by six courses of TMZ monotherapy, improved overall survival in patients with good performance status and age up to 70 years, and is currently the gold standard of care. ${ }^{48}$ Several targeted therapies have been evaluated in clinical trials, however currently not implemented in standard care. ${ }^{9}$ Clinical trials have demonstrated that hypofractionated radiation therapy with or without TMZ, or TMZ alone if $\mathrm{O}^{6}$-methylguanine-DNA methyl transferase (MGMT) promoter is methylated, are beneficial treatment options in elderly patients. ${ }^{10-12}$ Best supportive care may be an appropriate approach in the elderly and very frail patients, particularly in patients with multifocal or large tumours. ${ }^{4}$ Elderly patients, patients with poor performance status and patients lacking histological confirmation of the diagnosis, are excluded from most clinical trials. This may result in selection bias and impact survival rates.

We aimed to determine overall survival from glioblastoma in an unselected cohort of consecutive patients diagnosed with glioblastoma during an 8-year period in a geographically defined area of Western Norway. Furthermore, we analysed clinical and radiological characteristics associated with treatment approach, and the association between treatment intensity and survival.

\section{METHODS}

This was a population-based, retrospective cohort study of patients diagnosed with glioblastoma between 1 January 2007 and 31 December 2014. Patients aged 18 years or older, diagnosed with International Classification of Diseases 10th Revision code C71 (malignant neoplasm of brain) or C72 (malignant neoplasm of spinal cord, cranial nerves and other parts of central nervous system) in the Western region of Norway, were identified through electronic medical records. Both patients with histologically verified glioblastoma and patients where the glioblastoma diagnosis was based solely on typical MRI characteristics were enrolled. Patients from outside the region and patients with recurrent glioma, synchronous malignancies or lack of informed consent were excluded. During the study period, the predefined geographical region served a population of approximately $1020000 .^{13}$ All hospitals provided medical treatment and supportive care to patients with glioblastoma. Neurosurgical treatment of patients with brain tumour was centralised to one hospital, while radiation therapy was centralised to two hospitals in the region.

Demographics and patient characteristics were identified (table 1). Time of diagnosis was defined as the date of the first MRI detecting the primary brain tumour. The follow-up period was at least 5 years, or until death. We defined patients aged 70 years and older as elderly, based on the cut-off value in relevant studies and clinical practice in the region. ${ }^{1014-17}$ Comorbidity was classified according to the Charlson Comorbidity Index. ${ }^{18} \mathrm{We}$ registered any cognitive impairment described by clinicians, regardless of severity and causation. Radiological characteristics were obtained from MRI reports. MGMT promoter methylation and IDH (Isocitrate dehydrogenase) mutation analyses were not implemented as routine analysis in the study period.

Information regarding primary treatment, complications and survival were collected from medical records. Surgery was categorised into resection, biopsy and no surgical intervention. Primary CRT was categorised into full intensive treatment according to the Stupp protocol, less intensive CRT and best supportive care. Treatment according to the Stupp protocol was defined as a delivered radiation dose of 60 Gy (and optional additional boost), concomitant TMZ throughout the entire radiation therapy period and at least one out of six planned TMZ monotherapy courses fulfilled, in concordance with a previous and comparable study. ${ }^{19}$ Less intensive CRT was further classified into (i) full-course radiation therapy (60 Gy in 2 Gy fractions) and TMZ to a lesser extent than the Stupp protocol, (ii) short-course radiation therapy with concomitant TMZ and at least one monotherapy TMZ course, (iii) short-course radiation therapy with TMZ to a lesser extent or no TMZ and (iv) TMZ monotherapy without radiation therapy.

Adverse events and complications including infections, bone marrow suppression grade 3-4 according to Common Terminology Criteria for Adverse Events (CTCAE) V.5.0, venous thromboembolism, epileptic seizures and osteoporosis (defined as low-energy fracture or bone density below $-2.5 \mathrm{SD}$ measured by bone density scan) were identified. We calculated survival rates from time of diagnosis, and defined long-term survival as survival of $>5$ years.

\section{Patient and public involvement}

Patients and the public were not involved in this study.

\section{Statistics}

We used $\chi^{2}$ test or Fisher's exact test as appropriate for categorical variables. For continuous variables, we used a t-test for normally distributed data, otherwise the MannWhitney $\mathrm{U}$ test was used. Verification of normality was done by quantile-quantile plots. Clinical and radiological characteristics and their associations with treatment approach were analysed using binomial logistic regression. We applied the univariate and multivariate Cox proportional hazards regression models to evaluate the effect of treatment on overall survival. Cox proportional hazard assumption was tested for all variables. Clinical and radiological variables considered reasonably likely to influence the management approach and outcome were included in the models. Survival probabilities were calculated using a Kaplan-Meier plot and groups compared by log-rank test. Two-sided $p$ values $<0.05$ were considered statistically significant. Statistical analyses were performed with IBM SPSS Statistics V.24 (SPSS, Chicago, Illinois, USA). 
Table 1 Patient and tumour characteristics in adults diagnosed with glioblastoma between January 2007 and December 2014

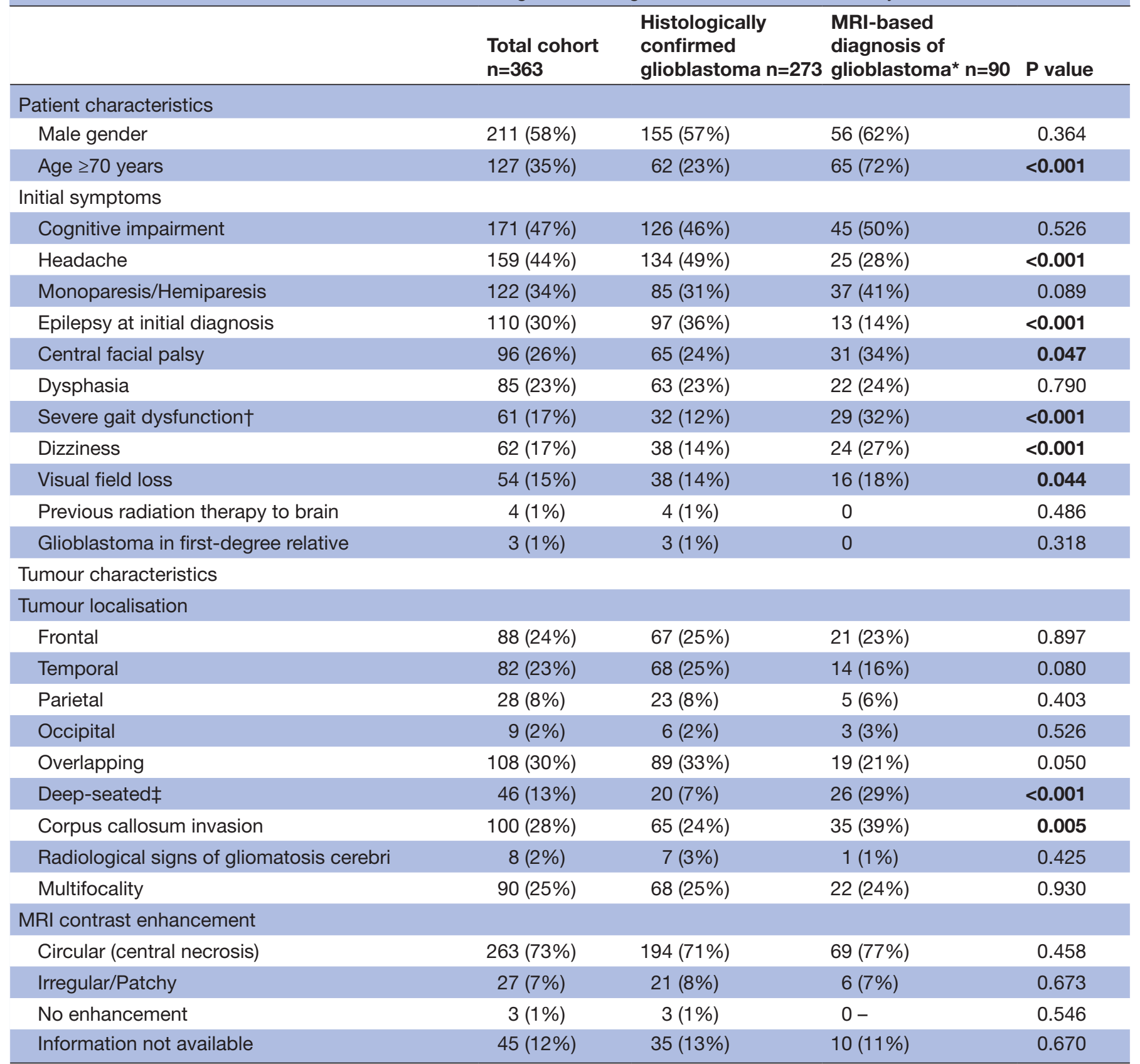

Results presented in absolute numbers and \% of total. Comparison between groups (histologically confirmed glioblastoma and MRI-based glioblastoma diagnosis) was performed by $\chi^{2}$ test (Fisher's exact test when expected cell count $<5$ ).

Significant $\mathrm{p}$ values are in bold.

*Highly suspected glioblastoma based on typical MRI characteristics, biopsy not performed.

†Inability to walk without support.

$\ddagger$ Thalamus, basal ganglia, internal capsule, splenium corpus callosum, mesencephalon, brain stem and cerebellum.

\section{RESULTS}

We identified 381 patients diagnosed with glioblastoma in the predefined geographical region between 1 January 2007 and 31 December 2014. From these, 16 patients were excluded according to exclusion criteria (non-resident $(\mathrm{n}=1)$, previous low-grade or anaplastic glioma $(\mathrm{n}=10)$, synchronous cancer $(n=4)$ and lack of informed consent $(\mathrm{n}=1))$. One patient was lost to follow-up and one patient was excluded due to disproved glioblastoma diagnosis by autopsy. The remaining 363 patients diagnosed with glioblastoma in the predefined period were included. None of these participated in clinical trials. Histological confirmation of the diagnosis was lacking in 90 patients (24.8\%), including two patients with non-representative biopsies, in whom the diagnosis was based on typical MRI characteristics. Among the 127 patients aged over 70 years, 65 (51.2\%) lacked histological confirmation of the diagnosis, compared with 25 of the 236 patients $(10.6 \%)$ aged under 70 years. 


\section{Tumour and patient characteristics}

Median age at the time of diagnosis was 64.6 years (range 18.1-94.9). Median age in patients with histologically confirmed glioblastoma was 61.5 years (range 18.1-86.1), compared with 77.0 years (range 35.0-94.9) in patients with an MRI-based diagnosis $(\mathrm{p}<0.0001)$. Mean Charlson Comorbidity Index was 3.9 (SD 1.4) in patients with histologically confirmed glioblastoma, compared with 5.7 (SD 1.6) in patients with an MRI-based diagnosis $(p<0.0001)$. Male/female ratio was 1.39 . Additional patient and tumour characteristics are outlined in table 1.

Headache and epilepsy were more frequent in patients with histologically confirmed glioblastoma compared with patients with an MRI-based diagnosis, while dizziness and gait dysfunction were more frequent among patients with an MRI-based diagnosis. Classification of performance status by validated screening tools (eg, Eastern Cooperative Oncology Group and Karnofsky score) was not applicable due to insufficient documentation of performance status in the medical records.

\section{Treatment and complications}

Surgical resection was performed in 221 of 363 patients $(60.9 \%)$. Radiation therapy was given to 323 patients $(89.0 \%)$, where full-course radiation therapy (60 Gy or $60 \mathrm{~Gy}$ with additional boost) was planned or commenced in 218 patients. Among these 218 patients, 14 (6.4\%) had the treatment cancelled $(n=1)$, discontinued $(n=5)$ or converted to a short-course regimen $(n=8)$. Change in radiation therapy plan was due to poor general condition or rapid clinical deterioration $(\mathrm{n}=11)$, patient preferences $(n=2)$ or acute complications $(n=1)$. Furthermore, 120 patients were allocated to short-course radiation therapy. Among these, the treatment was cancelled $(n=13)$ or discontinued $(\mathrm{n}=11)$ in 24 patients $(20.0 \%)$, due to poor general condition $(n=14)$, acute complications $(n=7)$ or patient preferences $(n=3)$.

CRT according to the Stupp protocol was prepared or commenced in 185 patients, while the treatment plan was changed or prematurely interrupted in 28 of these $(15.1 \%)$. Consequently, 157 patients $(43.3 \%$ of the total cohort) received CRT according to the Stupp protocol, including 11 patients without histological confirmation. Nine of these 11 patients had deep-seated tumours. In patients receiving adjuvant TMZ, regardless of radiation therapy dose, the mean number of TMZ courses was 4.6 (range 1-14). Nine of the patients who underwent resection were not eligible for CRT, due to complications, rapid progression or poor general condition. Among 236 patients aged under 70 years, $144(61.0 \%)$ received CRT according to the Stupp protocol, 85 patients $(36.0 \%)$ received less intensive CRT and 7 patients $(3.0 \%)$ received best supportive care. In the cohort of 127 patients aged over 70 years, $13(10.2 \%)$ received CRT according to the Stupp protocol, 82 patients $(64.6 \%)$ received less intensive CRT and 32 patients $(25.2 \%)$ received best supportive care.

Associations between patient and tumour characteristics and treatment approach are presented in table 2. Elderly patients, patients with multifocal or deep-seated tumours and patients with higher comorbidity burden were less likely to undergo surgical resection, according to adjusted logistic regression analyses. Elderly patients, patients with cognitive impairment, patients with increasing comorbidity burden and females were less likely to receive CRT according to the Stupp protocol.

In total, 188 patients $(51.8 \%)$ had at least one epileptic seizure, the majority at the time of diagnosis.

Table 2 Associations between patient and tumour characteristics and treatment approach in 363 patients diagnosed with glioblastoma between January 2007 and December 2014

\begin{tabular}{|c|c|c|c|c|c|c|c|c|}
\hline & \multicolumn{4}{|c|}{ No resection } & \multicolumn{4}{|c|}{ CRT less intensive than Stupp protocol† } \\
\hline & \multicolumn{2}{|c|}{ Unadjusted } & \multicolumn{2}{|c|}{ Adjusted } & \multicolumn{2}{|c|}{ Unadjusted } & \multicolumn{2}{|c|}{ Adjusted } \\
\hline & OR & (95\% Cl) & OR & $(95 \% \mathrm{Cl})$ & OR & $(95 \% \mathrm{Cl})$ & OR & $(95 \% \mathrm{Cl})$ \\
\hline Female gender & 1.1 & (0.7 to 1.6$)$ & 1.0 & (0.6 to 1.7$)$ & 1.7 & $(1.1 \text { to } 2.5)^{*}$ & 1.8 & $(1.1 \text { to } 3.1)^{*}$ \\
\hline Age $\geq 70$ years & 4.5 & $(2.9 \text { to } 7.2)^{\star \star \star}$ & 3.0 & $(1.5 \text { to } 6.3)^{\star *}$ & 13.7 & $(7.3 \text { to } 25.8)^{\star \star \star}$ & 5.1 & $(2.2 \text { to } 11.8)^{\star \star \star}$ \\
\hline Cognitive impairment & 1.2 & (0.8 to 1.8$)$ & 1.2 & (0.8 to 2.1$)$ & 1.8 & $(0.6 \text { to } 1.7)^{* *}$ & 1.8 & $(1.1 \text { to } 3.0)^{*}$ \\
\hline $\begin{array}{l}\text { Charlson Comorbidity } \\
\text { Index }\end{array}$ & 1.5 & $(1.3 \text { to } 1.8)^{\star \star \star}$ & 1.3 & $(1.0 \text { to } 1.6)^{*}$ & 2.2 & $(1.8 \text { to } 2.6)^{\star \star \star}$ & 1.6 & $(1.2 \text { to } 2.0)^{\star \star \star}$ \\
\hline Multifocal tumour & 1.9 & $(1.2 \text { to } 3.1)^{\star \star}$ & 2.6 & $(1.5 \text { to } 4.6)^{*}$ & 1.2 & (0.7 to 1.9$)$ & 1.6 & (0.9 to 2.9 ) \\
\hline Deep-seated tumourł & 7.3 & $(3.5 \text { to } 15.3)^{\star \star \star}$ & 10.0 & $(4.4 \text { to } 22.3)^{\star \star \star}$ & 1.4 & (0.7 to 2.6$)$ & 1.5 & (0.7 to 3.2$)$ \\
\hline
\end{tabular}

Significant $p$ values are in bold.

${ }^{*}$ Two-sided $p$ values $<0.05$ were considered statistically significant; ${ }^{*} p<0.05 ;{ }^{* *} p<0.01 ;{ }^{* * *} p<0.001$; results not marked by an asterisk are not significant.

†Stupp protocol=radiation therapy 60 Gy in 2 Gy fractions (delivered), fulfilled concomitant TMZ and fulfilled at least one out of six planned TMZ monotherapy courses.

$\ddagger$ Thalamus, basal ganglia, internal capsule, splenium corpus callosum, mesencephalon, brain stem and cerebellum.

CRT, chemoradiotherapy; TMZ, temozolomide. 
A
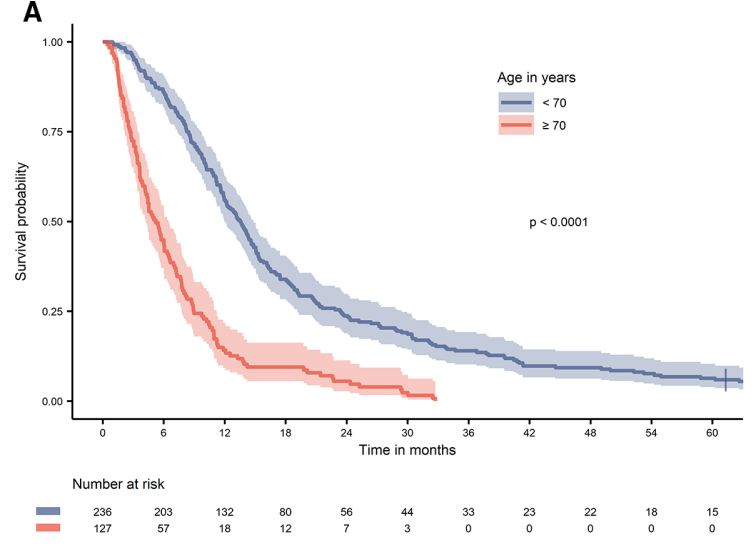

。

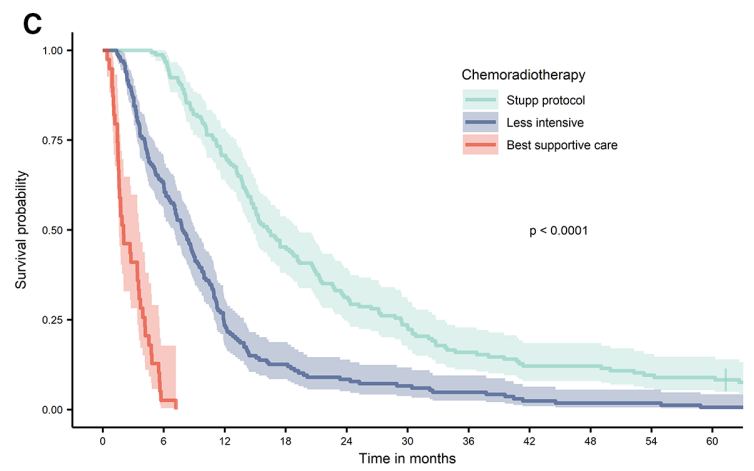

$=\begin{gathered}\text { Number at risk } \\ 157 \\ 167 \\ 39\end{gathered}$
B
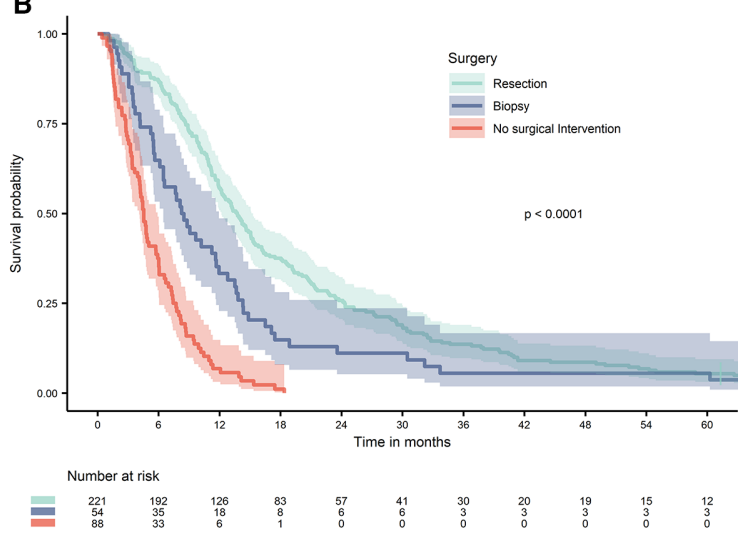

D

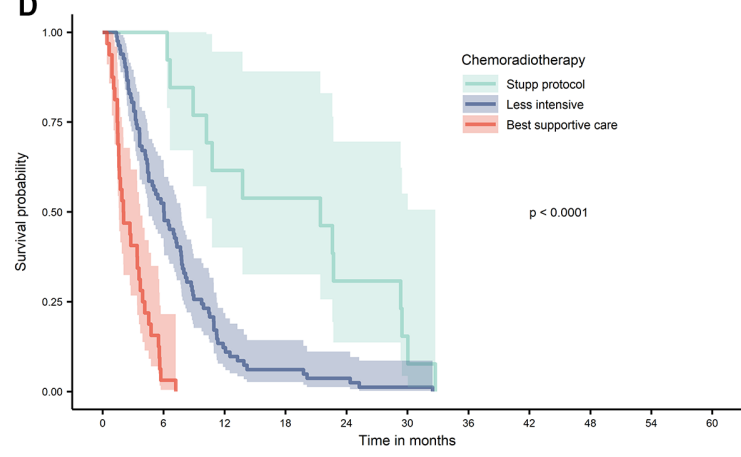

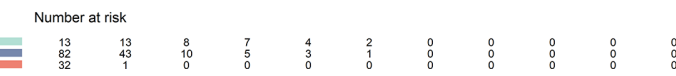

Figure 1 Overall survival in 363 adults diagnosed with glioblastoma between January 2007 and December 2014. (A) Survival by age. (B) Survival by surgical treatment. (C) Survival by chemoradiotherapy. (D) Survival by chemoradiotherapy in patients aged 70 years or older. Stupp protocol is here defined as completed radiation therapy in total dose of 60 Gy in 2 Gy fractions, concomitant temozolomide in the entire radiation therapy period and completed at least one out of six planned temozolomide monotherapy courses. Cumulative survival in months with $95 \% \mathrm{Cl}$ bands. Groups compared with log-rank test.

Venous thromboembolism (deep venous thrombosis of extremity, pulmonary embolism or sinus vein thrombosis) occurred in 75 patients $(20.7 \%)$, while 26 patients $(7.2 \%)$ were diagnosed with osteoporosis. Among 247 patients receiving initial chemotherapy, CTCAE grade 3-4 bone marrow suppression, that is, platelet count $<50.0 \times 10^{9} / \mathrm{L}$ and $/$ or neutrophil count $<1.0 \times 10^{9} / \mathrm{L}$, occurred in 37 patients $(15.0 \%)$. Fiftyeight patients $(23.5 \%)$ had bacterial or viral infections, while 11 patients $(4.5 \%)$ experienced septicaemia or neutropenic fever.

\section{Survival}

Median overall survival was 10.2 months (95\% CI 9.1 to 11.3 months). One-year, 2-year, 3-year and 5 -year survival rates were $41.3 \%, 17.3 \%, 9.1 \%$ and $4.1 \%$, respectively. Median survival among patients with histologically confirmed glioblastoma was 12.6 months (95\% CI 11.4 to 13.8 ), compared with 4.5 months (95\% CI 4.0 to 5.1) in patients with an MRIbased diagnosis $(\mathrm{p}<0.0001)$. In total, 354 of the 363 included patients $(97.5 \%)$ died during the study period, and one patient was lost to follow-up. KaplanMeier curves of survival according to age, surgery and CRT are presented in figure 1. Median survival in patients aged under 70 years was 13.5 months (95\% CI 12.1 to 14.9 ), compared with 5.2 months (95\% CI 4.1 to 6.3 ) in patients aged over 70 years. Median survival in patients who underwent resection was 13.7 months (95\% CI 12.1 to 15.4 ), compared with 8.3 months (95\% CI 6.6 to 9.9) for those who underwent biopsy, and 4.5 months (95\% CI 4.0 to 5.1) in patients with no surgical intervention. Median survival in patients receiving CRT according to the Stupp protocol was 16.3 months (95\% CI 14.1 to 18.5), compared with 7.9 months (95\% CI 6.7 to 9.0) and 2.0 months (95\% CI 0.9 to 3.2) in patients treated with less intensive CRT or best supportive care, respectively. In patients aged over 70 years and receiving CRT according to the Stupp protocol, median survival was 21.4 months (95\% CI 7.5 to 35.3 ), compared with 6.0 months (95\% CI 4.7 to 7.7) and 2.0 months (95\% CI 0.7.3.4) in those treated with less intensive CRT or best supportive care. Among 157 patients receiving CRT according to the Stupp protocol, 49 patients $(31.2 \%)$ survived for $>2$ years, and 14 patients $(8.9 \%)$ survived for $>5$ years.

Univariate and multivariate Cox proportional hazards regression models of overall survival are presented in table 3. Resection compared with no 
Table 3 Univariate and multivariate Cox regression analysis of overall survival in 363 patients diagnosed with glioblastoma between January 2007 and December 2014

\begin{tabular}{|c|c|c|c|c|c|c|}
\hline \multirow[b]{2}{*}{ Variables } & \multicolumn{3}{|c|}{ Univariate analysis } & \multicolumn{3}{|c|}{ Multivariate analysis } \\
\hline & HR & $(95 \% \mathrm{Cl})$ & $P$ value & HR & $(95 \% \mathrm{Cl})$ & $P$ value \\
\hline Female gender & 1.04 & (0.784 to 1.29$)$ & 0.71 & 0.91 & (0.73 to 1.14$)$ & 0.40 \\
\hline Cognitive impairment & 1.25 & (1.01 to 1.54$)$ & 0.04 & 1.06 & (0.84 to 1.32$)$ & 0.64 \\
\hline Charlson Comorbidity Index & 1.44 & (1.34 to 1.54$)$ & $<0.001$ & 1.22 & (1.10 to 1.35$)$ & $<0.001$ \\
\hline Multifocality & 1.53 & (1.20 to 1.95$)$ & $<0.01$ & 1.42 & (1.09 to 1.84$)$ & $<0.01$ \\
\hline \multicolumn{7}{|l|}{ Surgical treatment } \\
\hline No resection & Ref & & & Ref & & \\
\hline Resection & 0.39 & (0.31 to 0.48$)$ & $<0.001$ & 0.61 & (0.47 to 0.79$)$ & $<0.001$ \\
\hline \multicolumn{7}{|l|}{ Chemoradiotherapy (CRT) } \\
\hline
\end{tabular}

$\mathrm{HR}, 95 \% \mathrm{Cl}$ and $\mathrm{p}$ values calculated by univariate and multivariate Cox proportional hazards regression model. $\mathrm{P}$ values $<0.05$ were considered statistically significant.

Significant $p$ values are in bold.

*Thalamus, basal ganglia, internal capsule, splenium corpus callosum, mesencephalon, brain stem and cerebellar vermis.

†Radiation therapy 60 Gy in 2 Gy fractions (delivered), fulfilled concomitant TMZ and fulfilled at least one out of six planned TMZ monotherapy courses.

TMZ, temozolomide.

resection was strongly associated with improved overall survival according to multivariate analyses (HR 0.61, p<0.001). CRT according to the Stupp protocol (HR 0.09, $\mathrm{p}<0.001)$ and less intensive CRT
(HR 0.17, p<0.001) were strongly associated with better outcomes.

An alluvial diagram visualises the consecutive treatment modalities and the association with median

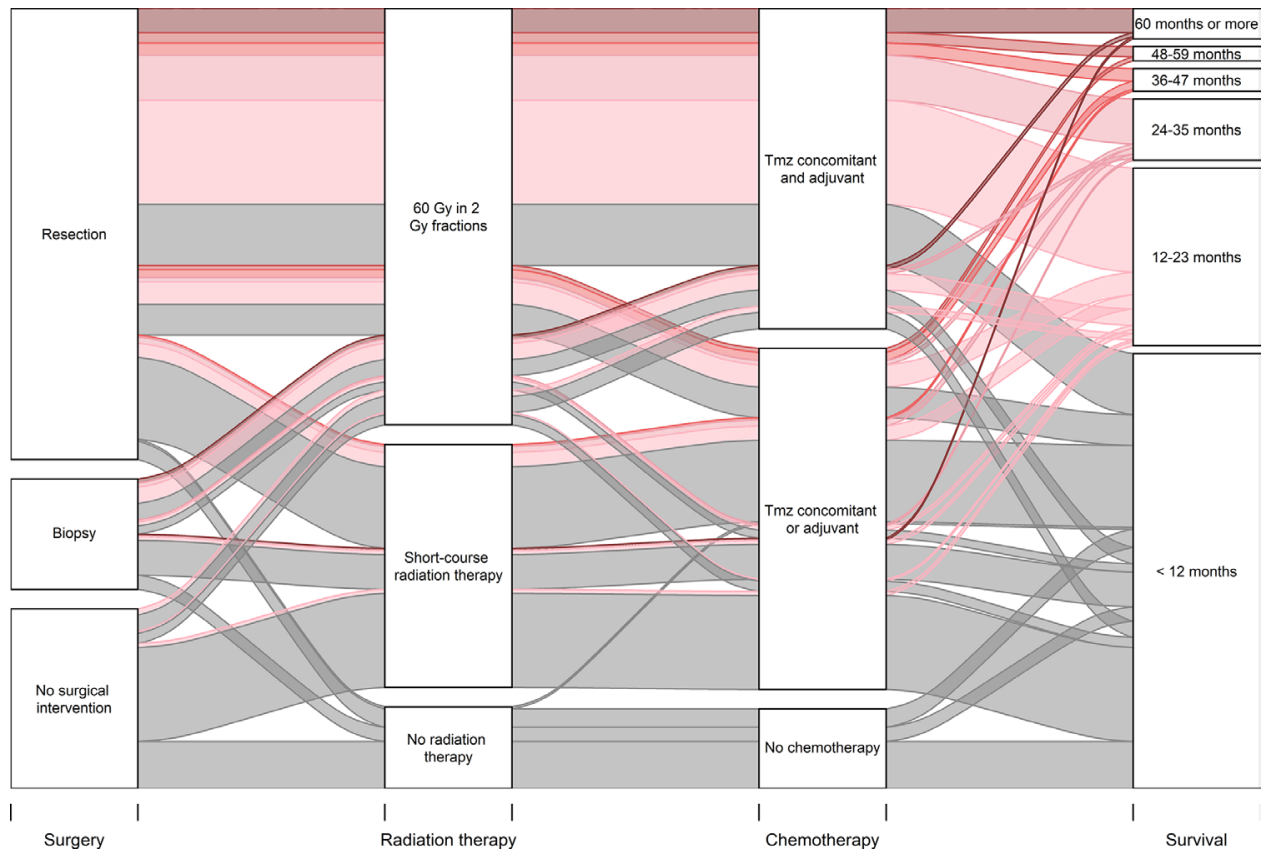

Figure 2 Alluvial diagram visualising associations between combination of treatment modalities and median survival in an unselected cohort of 363 patients diagnosed with glioblastoma between January 2007 and December 2014. The width of the curves represents the absolute number of patients. The colours of the curves correspond to median survival in months. TMZ, temozolomide. 
survival (figure 2). In the whole cohort, 15 patients $(4.1 \%)$ achieved long-term survival of $>5$ years. Twelve of these patients underwent surgical resection, whereas three had a biopsy alone. Moreover, 14 out of 15 long-term surviving patients completed the Stupp protocol, while one patient received hypofractionated radiation therapy followed by TMZ monotherapy. All 15 long-term surviving patients completed at least six maintenance TMZ courses (range six to nine).

\section{DISCUSSION}

Median overall survival in our cohort of 363 consecutive patients diagnosed with glioblastoma was approximately 10 months. Surgical resection and full-course CRT were strongly associated with improved survival, as demonstrated by multivariate Cox regression. However, only twothirds of the patients underwent resection, and less than half of the patients received CRT according to the Stupp protocol. Age over 70 years was strongly associated with less intensive treatment, both surgery and CRT. Irrespective of age, those who received treatment according to the Stupp protocol, had a favourable prognosis with median survival and long-term survival rates comparable to those observed in clinical trials. Survival was considerably worse in elderly patients and patients receiving less intensive treatment. A significant number of patients received best supportive care only, thus the overall survival was poorer in this population-based study compared with results from clinical trials.

Histological confirmation of the diagnosis was lacking in approximately $25 \%$ of the patients in our cohort. There are limited real-world data describing the frequency of omitting biopsy in patients with a high suspicion of glioblastoma according to MRI. A previous Norwegian study reported that $12 \%$ of the patients diagnosed with glioblastoma had a diagnosis based solely on radiological pattern or autopsy. ${ }^{20}$ Conversely, an English population-based study reported that $<10 \%$ of patients aged under 70 years, and $40 \%$ of patients aged over 70 years, lacked histological confirmation of the diagnosis, comparable to our findings. ${ }^{21}$ We found that patients with MRI-based diagnoses were older and had a higher comorbidity burden, and they more often had deep-seated tumours. In addition, they more commonly presented with dizziness and gait disturbances, which are vague and often slowly progressing symptoms that may have led to a delay in diagnosis compared with patients presenting with epileptic seizure or headache. It is reasonable to assume that established experiences and traditions among clinicians may influence the choice of intervention, for example, emphasising the risk of complications related to neurosurgery in elderly or frail patients, and patients with deepseated tumours. The dismal prognosis of patients not undergoing resection is another possible contributing factor to the choice of this managment. A further reason may be the improvement of MRI techniques, including perfusion-weighted imaging and diffusion-weighted MRI, facilitating the distinction of glioblastoma from other intracranial lesions. ${ }^{22}{ }^{23}$ However, in order to increase the diagnostic accuracy, biopsy should also be recommended in patients considered unlikely to benefit from resection, when considered feasible and safe.

Resection was performed in $61 \%$ of the patients in this cohort, in line with the aforementioned study from England..$^{21}$ However, the resection rate was lower than reported in other previous population-based studies, where $74 \%$ of patients underwent resection. ${ }^{319}$ A possible explanation is our inclusion of patients with an MRI-based glioblastoma diagnosis, and a higher number of patients with deep-seated tumours. Patients who underwent resection had a significantly better survival than those who underwent biopsy or no surgical intervention.

Nearly $90 \%$ of the patients in our cohort received radiotherapy, the majority in combination with TMZ. Multivariate Cox regression, with adjustment for age and other clinical variables, demonstrated improved overall survival in patients receiving CRT according to the Stupp. However, less than half of the patients received CRT according to the Stupp protocol, similar to the findings of Lwin et al. ${ }^{19}$ We assume that the frequency of elderly patients, patients with a significant comorbidity burden and patients with extensive symptoms including cognitive impairment, influence the choice of therapeutic intensity and the capacity of patients to complete commenced treatment. Patients aged over 70 years received less intensive treatment compared with younger patients, in concordance with previous studies of elderly patients with glioblastoma. ${ }^{31415} 21$

Median overall survival in our cohort was approximately 10 months, comparable with results from previous population-based studies with median survival ranging from 6.1 to 15.3 months. ${ }^{3}{ }^{19-21} 2425$ A 5-year survival rate of approximately $4 \%$ was equal to that reported in large population-based materials. ${ }^{121}$ A recent systematic review reported a superior median overall survival of 15.6 months in the post-Stupp era. ${ }^{26}$ However, nearly onethird of the studies included in this review article were clinical trials, with an expected superiority in survival rates compared with population-based materials. In our cohort, outcome was considerably better in patients receiving CRT according to the Stupp protocol, with a median survival of approximately 16 months and a 5 -year survival rate of $8.9 \%$. This was in line with the results from the randomised clinical trial by Stupp et al, where median survival in the CRT arm was 14.6 months, and 5-year survival was $9.8 \% .{ }^{827}$ Our results highlight the gap between the survival rates reported from clinical studies and those observed in a real-world setting.

Median overall survival in patients aged over 70 years was 5.2 months in our cohort, in line with previous population-based studies where median survival ranged from <3-4 months. ${ }^{14151721}$ Survival in elderly patients in our cohort was strongly associated with a CRT treatment approach, and ranged from 2 months in patients receiving best supportive care to 21 months in patients receiving 
CRT according to the Stupp protocol. This was comparable to results from previous population-based studies in elderly patients. ${ }^{38}$ As expected, median overall survival in elderly patients was lower in our unselected cohort than that demonstrated in prospective clinical trials, where median survival ranged from 5.2 to 9.6 months depending on CRT. ${ }^{10-12} 16$ A recent Cochrane analysis concluded that CRT improved survival compared with radiation therapy alone in elderly patients capable of selfcare. ${ }^{29}$ The improved survival in elderly patients receiving combined CRT, both in our cohort and previous studies, demonstrates a potential benefit from intensive treatment in this group. ${ }^{3} 112830$ A disregard of this issue may risk potentially undertreating elderly patients. Nevertheless, in patients of advanced age, or suffering from extensive disabilities, best supportive care may be an appropriate approach.

As concerns the methodology of our study, we regard the population-based design as a strength. The long-term follow-up of an unselected cohort provides knowledge on treatment and survival from glioblastoma, including the establishment of long-term survival rates, and the inclusion period ensured that all included patients were diagnosed with glioblastoma after the implementation of the current standard treatment. Other strengths were the low dropout rate of only one patient $(0.3 \%)$, and the detailed clinical information on treatment and complications available in all patients within a common patient record system throughout the region. Among the limitations of the study was the lack of molecular analyses. Furthermore, performance status was not sufficiently described in medical records and not applicable to validated screening tools. To counteract this, comorbidity burden, cognitive impairment and gait dysfunction were included in the analyses. In addition, surgical resection was not classified into degree of resection; hence, the survival curves did not differentiate between macroscopic complete and partial resection. The inclusion of patients with MRIbased diagnosis can be considered both a disadvantage and an advantage. To reduce the risk of incorrect inclusion of patients without glioblastoma, we included only patients when clinicians and radiologists unequivocally considered glioblastoma the most likely diagnosis. Even though biopsy is highly recommended and is standard of care, it is not always considered feasible and safe. Therefore, the inclusion of these patients provides knowledge on the diagnostic approach and survival of all patients with highly suspected glioblastoma based on MRI.

In conclusion, the prognosis of glioblastoma was considerably worse in a real-world setting compared with results from clinical trials. In patients receiving treatment according to the Stupp protocol, survival rates were comparable to that achieved in clinical trials. Multivariate Cox regression demonstrated that both resection and CRT were strongly associated with better outcome. However, only two-thirds of the patients in our cohort underwent resection, and less than half of the patients received treatment according to the Stupp protocol. Our results point towards a substantial risk of undertreating patients with glioblastoma, especially in elderly patients, and a potential benefit from choosing a more aggressive treatment approach.

\section{Author affiliations}

${ }^{1}$ Department of Clinical Medicine, University of Bergen Faculty of Medicine and Dentistry, Bergen, Norway

${ }^{2}$ Department of Haematology and Oncology, Stavanger University Hospital, Stavanger, Norway

${ }^{3}$ Department of Oncology and Medical Physics, Haukeland Universitetssjukehus, Bergen, Norway

${ }^{4}$ Department of Clinical Science, University of Bergen Faculty of Medicine and Dentistry, Bergen, Norway

${ }^{5}$ Department of Neurosurgery, Haukeland University Hospital, Bergen, Norway

${ }^{6}$ Department of Neurology, Stavanger University Hospital, Stavanger, Norway

Acknowledgements The authors would like to thank Anastasia Ushakova at Stavanger University Hospital for support in statistical analyses, and Joanna Haynes at Stavanger University Hospital for proofreading the article.

Contributors All coauthors have contributed to the design of the work and, are accountable for all its aspects. LSB, OF and EF initiated the study and were responsible for study design and data collecting. LSB was responsible for statistical analyses, contributed by $\mathrm{OF}, \mathrm{BG}, \mathrm{RM}$ and $\mathrm{EF}$, with support from statistician AU. All coauthors participated in the interpretation of the results. LSB and OF designed the figures. LSB drafted the initial manuscript, and all coauthors reviewed and edited the paper. All coauthors approved the final version of the manuscript.

Funding The authors have not declared a specific grant for this research from any funding agency in the public, commercial or not-for-profit sectors.

Competing interests None declared.

Patient consent for publication Not required.

Ethics approval The study was approved by the Regional Committee for Medical and Health Research Ethics (no. 2014/1931). Informed consent was obtained from patients alive at the time of inclusion. A waiver of consent was approved for deceased patients.

Provenance and peer review Not commissioned; externally peer reviewed.

Data availability statement № data are available.

Open access This is an open access article distributed in accordance with the Creative Commons Attribution Non Commercial (CC BY-NC 4.0) license, which permits others to distribute, remix, adapt, build upon this work non-commercially, and license their derivative works on different terms, provided the original work is properly cited, appropriate credit is given, any changes made indicated, and the use is non-commercial. See: http://creativecommons.org/licenses/by-nc/4.0/.

ORCID iD

Line Sagerup Bjorland http://orcid.org/0000-0003-3043-7804

\section{REFERENCES}

1 Ostrom QT, Gittleman H, Truitt G, et al. CBTRUS statistical report: primary brain and other central nervous system tumors diagnosed in the United States in 2011-2015. Neuro Oncol 2018;20:iv1-86.

2 Wen PY, Huse JT. 2016 World Health organization classification of central nervous system tumors. Continuum 2017;23:1531-47.

3 Hansen S, Rasmussen BK, Laursen RJ, et al. Treatment and survival of glioblastoma patients in Denmark: the Danish neuro-oncology registry 2009-2014. J Neurooncol 2018;139:479-89.

4 Weller M, van den Bent M, Hopkins K, et al. EANO guideline for the diagnosis and treatment of anaplastic gliomas and glioblastoma. Lancet Oncol 2014;15:e395-403.

5 Saini J, Kumar Gupta P, Awasthi A, et al. Multiparametric imagingbased differentiation of lymphoma and glioblastoma: using T1perfusion, diffusion, and susceptibility-weighted MRI. Clin Radiol 2018;73:986.e7-986.e15.

6 Suh $\mathrm{CH}$, Kim HS, Jung SC, et al. Perfusion MRI as a diagnostic biomarker for differentiating glioma from brain metastasis: a systematic review and meta-analysis. Eur Radiol 2018;28:3819-31. 
7 Sulman EP, Ismaila N, Armstrong TS, et al. Radiation therapy for glioblastoma: American Society of clinical oncology clinical practice guideline endorsement of the American Society for radiation oncology guideline. J Clin Oncol 2017;35:361-9.

8 Stupp R, Mason WP, van den Bent MJ, et al. Radiotherapy plus concomitant and adjuvant temozolomide for glioblastoma. $N$ Engl $J$ Med Overseas Ed 2005;352:987-96.

9 Mohtashami E, Shafaei-Bajestani N, Mollazadeh H, et al. The current state of potential therapeutic modalities for glioblastoma multiforme: a clinical review. Curr Drug Metab 2020;21:564-78.

10 Malmström A, Grønberg BH, Marosi C, et al. Temozolomide versus standard 6-week radiotherapy versus hypofractionated radiotherapy in patients older than 60 years with glioblastoma: the Nordic randomised, phase 3 trial. Lancet Oncol 2012;13:916-26.

11 Perry JR, Laperriere N, O'Callaghan CJ, et al. Short-Course radiation plus temozolomide in elderly patients with glioblastoma. $N$ Engl $J$ Med 2017;376:1027-37.

12 Wick W, Platten M, Meisner C, et al. Temozolomide chemotherapy alone versus radiotherapy alone for malignant astrocytoma in the elderly: the NOA-08 randomised, phase 3 trial. Lancet Oncol 2012;13:707-15.

13 Statistics Norway. 2020:Population and area (M):2007-20.

14 Coate L, McNamara MG, Lwin Z, et al. Glioblastoma treatment in the elderly in the temozolomide therapy era. Can J Neurol Sci 2014;41:357-62.

15 Gately L, Collins A, Murphy M, et al. Age alone is not a predictor for survival in glioblastoma. J Neurooncol 2016;129:479-85.

16 Minniti G, De Sanctis V, Muni R, et al. Hypofractionated radiotherapy followed by adjuvant chemotherapy with temozolomide in elderly patients with glioblastoma. J Neurooncol 2009;91:95-100.

17 Pretanvil J-A, Salinas IQ, Piccioni DE. Glioblastoma in the elderly: treatment patterns and survival. CNS Oncol 2017;6:19-28.

18 Charlson M, Szatrowski TP, Peterson J, et al. Validation of a combined comorbidity index. J Clin Epidemiol 1994;47:1245-51.

19 Lwin Z, MacFadden D, Al-Zahrani A, et al. Glioblastoma management in the temozolomide era: have we improved outcome? J Neurooncol 2013;115:303-10.
20 Rønning PA, Helseth E, Meling TR, et al. A population-based study on the effect of temozolomide in the treatment of glioblastoma multiforme. Neuro Oncol 2012;14:1178-84.

21 Brodbelt A, Greenberg D, Winters T, et al. Glioblastoma in England: 2007-2011. Eur J Cancer 2015;51:533-42.

22 Chiang IC, Kuo Y-T, Lu C-Y, et al. Distinction between high-grade gliomas and solitary metastases using peritumoral 3-T magnetic resonance spectroscopy, diffusion, and perfusion imagings. Neuroradiology 2004;46:619-27.

23 Radbruch A, Wiestler B, Kramp L, et al. Differentiation of glioblastoma and primary CNS lymphomas using susceptibility weighted imaging. Eur J Radiol 2013;82:552-6.

24 Teo M, Martin S, Owusu-Agyemang K, et al. A survival analysis of GBM patients in the West of Scotland pre- and post-introduction of the Stupp regime. Br J Neurosurg 2014;28:351-5.

25 Bruhn H, Strandéus M, Milos P. Improved survival of Swedish glioblastoma patients treated according to Stupp 2018;138:332-7.

26 Marenco-Hillembrand L, Wijesekera O, Suarez-Meade P, et al. Trends in glioblastoma: outcomes over time and type of intervention: a systematic evidence based analysis. $J$ Neurooncol 2020;147:297-307.

27 Stupp R, Hegi ME, Mason WP, et al. Effects of radiotherapy with concomitant and adjuvant temozolomide versus radiotherapy alone on survival in glioblastoma in a randomised phase III study: 5-year analysis of the EORTC-NCIC trial. Lancet Oncol 2009;10:459-66.

28 Rusthoven CG, Koshy M, Sher DJ, et al. Combined-Modality therapy with radiation and chemotherapy for elderly patients with glioblastoma in the temozolomide era: a national cancer database analysis. JAMA Neurol 2016;73:821-8.

29 Hanna C, Lawrie TA, Rogozińska E, et al. Treatment of newly diagnosed glioblastoma in the elderly: a network meta-analysis. Cochrane Database Syst Rev 2020;3:Cd013261.

30 Youssef M, Ludmir EB, Mandel JJ, et al. Treatment strategies for glioblastoma in older patients: age is just a number. $J$ Neurooncol 2019;145:357-64. 